\title{
Leyes que prohíben fumar en espacios cerrados en México
}

\author{
Raydel Valdés-Salgado, MS, MPH, (I) Érika Ávila-Tang, PhD, MHS, ${ }^{(I)}$ Frances A Stillman, EdD, (I) \\ Heather Wipfli, PhD, (I) Jonathan M Samet, MD, MS. ${ }^{(1)}$
}

\author{
Valdés-Salgado R, Ávila-Tang E, Stillman FA, \\ Wipfli H, Samet JM. \\ Leyes que prohíben fumar \\ en espacios cerrados en México. \\ Salud Publica Mex 2008;50 supl 3:S334-S342.
}

\section{Resumen}

Recientemente México aprobó una ley federal y otra en la capital del país que prohíben fumar en espacios cerrados. Estas medidas legislativas están en plena concordancia con las acciones propuestas en el Artículo 8 del Convenio Marco para el Control del Tabaco de la Organización Mundial de la Salud, que México ratificó en 2004. En este ensayo se revisa la evidencia científica que demuestra que la exposición al humo de tabaco causa enfermedades crónicas y agudas en niños y adultos no fumadores. Toda exposición al humo de tabaco es dañina, por lo tanto sólo la creación de espacios $100 \%$ libres de humo de tabaco, tal como lo plantean las nuevas leyes, constituye una intervención efectiva para proteger a la población. Este tipo de medidas se asocian con impactos positivos para la salud, como la reducción de la prevalencia de fumadores y del número de cigarrillos fumados diariamente, así como incremento en las tasas de cesación. En este texto, también proveemos evidencia de disminución de síntomas respiratorios agudos y de hospitalizaciones por infarto agudo del miocardio. Los principales argumentos de la industria tabacalera son desmentidos con ejemplos bien documentados de que no hay pérdidas económicas causadas por este tipo de medidas y de que la prohibición de fumar es aceptada por la población. Con las leyes aprobadas, el control del tabaco en México se ha fortalecido y entre los retos futuros están la implementación, evaluación y vigilancia del cumplimiento de la prohibición, así como otras leyes similares en los estados.

Palabras clave: tabaco; legislación; contaminación por humo de tabaco; México

\author{
Valdés-Salgado R, Ávila-Tang E, Stillman FA, \\ Wipfli H, Samet JM. \\ Laws that ban smoking \\ in indoor places in Mexico. \\ Salud Publica Mex 2008;50 suppl 3:S334-S342.
}

\begin{abstract}
Recently Mexico passed federal and state-level laws banning smoking in indoor spaces. These actions are totally in accordance with measures proposed in the WHO-FCTC's article 8 , ratified by Mexico in 2004 . This essay reviews scientific evidence that secondhand smoke causes both chronic and acute diseases among non smokers, children and adults. There is no safe level of exposure to secondhand smoke, so the only effective intervention to protect Mexican people from such harmful exposure is by promoting $100 \%$ smoke free indoor air, as the two new laws do. Total banning of smoking is associated with reduction in smoking prevalence, amount of cigarette smoked per day, and an increase in cessation rates. Reductions in acute respiratory symptoms and hospitalization of acute myocardial infarction have been also reported. Well documented examples show that main tobacco industry arguments against smoke free policy: negative economic impact and lack of support from the public, are unjustified. These two laws strengthen the Mexican tobacco control program. Next steps include their implementation, evaluation, enforcement of compliance and encouragement for similar laws at state level.
\end{abstract}

Key words: tobacco; legislation; tobacco smoke pollution, smoking cessation; Mexico

(I) Institute for Global Tobacco Control, Department of Epidemiology, Johns Hopkins Bloomberg School of Public Health, USA. 
$\mathrm{E}^{\mathrm{n}}$ los últimos meses México ha dado pasos muy significativos en su agenda para el control del tabaco, específicamente para proteger a la población de la exposición al humo de tabaco. En el ámbito federal se aprobó la Ley General para el Control del Tabaco, ${ }^{1}$ un documento en concordancia con las medidas propuestas en el Convenio Marco para el Control del Tabaco (CMCT) de la Organización Mundial de la Salud (OMS). En la capital del país se aprobaron importantes modificaciones a la ya existente Ley de Protección a la Salud de los No Fumadores en el Distrito Federal. ${ }^{2}$ En ambos casos millones de mexicanos serán beneficiados. El compromiso de México en esta lucha queda claro con ambas leyes y con el hecho de que a principios de 2007 se aplicó un nuevo impuesto directo a la venta de cigarrillos. ${ }^{3}$

Aunque estas prohibiciones de fumar en lugares cerrados son comprensibles desde el punto de vista de la salud pública, su puesta en práctica generalmente encuentra algunos obstáculos. En este ensayo se revisa tanto la evidencia científica que justifica que se proteja a la población de la exposición al humo de tabaco, como los principales argumentos que ha usado la industria tabacalera para impedir dicha medida. Para cada uno de esos argumentos se presenta evidencia que demuestra que los temores de pérdida económica estimulados por la industria, así como un posible rechazo a estas medidas por parte de la población son injustificados. Otros países ya se han declarado exitosamente libres de humo de tabaco y México también está en condiciones de lograrlo.

México es atractivo para la industria tabacalera por las potencialidades de mercado: una población de más de 100 millones de personas, donde el consumo diario es bajo (82\% de los fumadores, fuma 10 o menos cigarrillos al día) y donde las mujeres fuman considerablemente menos que los hombres. ${ }^{4}$ Estudios del consumo de cigarrillos entre adolescentes en 2003 y 2006 muestran que no hay diferencia entre hombre y mujeres, y que $28 \%$ de los no fumadores es susceptible de empezar a fumar en menos de un año. ${ }^{5}$ En este contexto, el entusiasmo por las dos leyes aprobadas, así como por cualquier otra medida antitabaco, está bien justificado.

\section{Exposición pasiva al humo de tabaco o humo de tabaco de segunda mano*}

Cuando personas que fuman comparten el espacio con quienes no fuman, estos últimos inhalan humo

\footnotetext{
* Humo de tabaco es el término general que usaremos en este texto en español para referirnos a lo que también se conoce como humo de tabaco de segunda mano (secondhand smoke, en inglés) o humo de tabaco ambiental (environmental tobacco smoke, en inglés).
}

de tabaco. El humo de tabaco es una mezcla de gases y partículas que resulta de la combinación del humo principal -exhalado por el fumador-y el llamado humo secundario, que es emitido por el cigarro directamente. ${ }^{6}$ En 1981 se publicó por primera vez acerca del exceso de mortalidad por cáncer de pulmón entre mujeres japonesas no fumadoras, pero cuyos esposos eran fumadores. ${ }^{7}$ Sin embargo, los efectos nocivos para la salud por la exposición al humo de tabaco ya habían sido foco de la investigación científica desde fines de los años sesenta, particularmente en niños ${ }^{8}$ y el informe del Cirujano General (CG) de Estados Unidos (EUA) de 1972 presentó evidencia sobre la posibilidad de efectos dañinos a la salud de los no fumadores. ${ }^{9}$

El aumento en el riesgo debido a inhalación de humo de tabaco se demostró primero en estudios de salud respiratoria de niños hijos de padres fumadores y posteriormente en estudios que revelaban mayor riesgo de cáncer de pulmón y cardiopatía coronaria en adultos no fumadores expuestos. Según la evidencia se fue acumulando, diversos grupos de expertos publicaron informes con conclusiones de gran peso político, como el informe de 1986 del CG Dr. Koop ${ }^{10}$ y la evaluación de riesgos llevada a cabo en 1992 por la Agencia de Protección Ambiental de EUA. ${ }^{11}$ Informes más recientes incluyen el del Comité Científico del Reino Unido sobre Tabaco, ${ }^{12}$ el de la Agencia de Protección Ambiental del Estado de California, ${ }_{13}^{13}$ una consulta internacional auspiciada por la OMS, ${ }^{14}$ un reporte del Centro Internacional de Investigaciones sobre el Cáncer ${ }^{15}$ y el Reporte del CG de EUA en $2006{ }^{16}$

La industria tabacalera busca crear dudas sobre los daños causados por el humo de tabaco y al respecto sus actividades en América Latina están bien documentadas. ${ }^{17}$ Sin embargo, los informes arriba mencionados ofrecen evaluaciones integrales de la evidencia y examinan los juicios de causalidad de las asociaciones basados en directrices para la interpretación de la evidencia. La determinación de que una asociación es causal es de importancia particular para las políticas, ya que posibilita la prevención mediante la reducción de exposiciones. Hoy no dudamos en decir que en los no fumadores, la exposición al humo de tabaco causa enfermedad y muerte prematura. El cuadro I sintetiza la evidencia actual que apoya la conclusión de que el humo de tabaco causa efectos dañinos agudos y crónicos en niños y en adultos.

En niños, la exposición al humo de tabaco causa bajo peso al nacer, enfermedades en las vías respiratorias inferiores, síntomas respiratorios crónicos, enfermedades del oído medio y reducción en la función pulmonar. ${ }^{10,18}$ Asimismo, se ha determinado que la exposición al humo de tabaco exacerba los episodios y síntomas del asma 


\section{Cuadro I}

Síntesis de los EFECTOS dAÑINOS A LA SALUd de NIÑOS Y ADULTOS POR EXPOSICIÓN AL HUMO de tABACO*

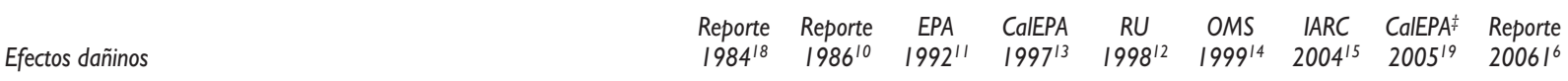

Niños

\begin{tabular}{|c|c|c|c|c|c|c|c|c|}
\hline Aumento en la prevalencia de síntomas respiratorios crónicos & A & A & $\mathrm{C}$ & $\mathrm{C}$ & C & $\mathrm{C}$ & $\mathrm{C}$ & $\mathrm{C}$ \\
\hline Disminución de la función pulmonar & A & A & A & A & & C & A & C \\
\hline Aumento en la aparición de enfermedades respiratorias agudas & A & A & A & $\mathrm{C}$ & & $\mathrm{C}$ & $\mathrm{C}$ & $\mathrm{C}$ \\
\hline Aumento en la aparición de enfermedades del oído medio & & A & $\mathrm{C}$ & $\mathrm{C}$ & C & $\mathrm{C}$ & $\mathrm{C}$ & C \\
\hline Aumento en la gravedad de episodios y síntomas de asma & & & C & $\mathrm{C}$ & & $\mathrm{C}$ & $\mathrm{C}$ & C \\
\hline Factor de riesgo de asma & & & $\mathrm{C}$ & $\mathrm{C}$ & & & $\mathrm{C}$ & $\mathrm{C}$ \\
\hline Factor de riesgo de síndrome de muerte súbita del lactante & & & & $\mathrm{C}$ & A & $\mathrm{C}$ & $\mathrm{C}$ & $\mathrm{C}$ \\
\hline
\end{tabular}

Adultos

Factor de riesgo de cáncer de pulmón en adultos C $\quad C \quad C \quad C$

C C C

Factor de riesgo de cáncer de mama para mujeres jóvenes,

principalmente premenopáusica

Factor de riesgo de cáncer de senos paranasales

Factor de riesgo de cardiopatía en adultos

C

Versión modificada del cuadro original publicado en: Samet JM, Wang SS. Environmental tobacco smoke. En: Lippmann M, ed. Environmental Toxicants. 2nd ed. New York: John Wiley \& Sons, 2000;319-375

* Sólo efectos causados por exposición al humo de tabaco (no incluyó relaciones de asociación)

$A=$ asociación

$\mathrm{C}=$ causa

y que es una causa de síndrome de muerte súbita del lactante. ${ }^{19}$

En los adultos, la exposición al humo de tabaco causa cáncer de pulmón y de los senos paranasales, así como cardiopatía coronaria, y posiblemente otros síntomas y enfermedades respiratorias, así como la disminución en la función pulmonar. Un informe presentado por el estado de California ha determinado que en mujeres jóvenes el humo de tabaco causa cáncer se mama. ${ }^{19}$ En muchas partes del mundo los hombres constituyen la mayoría de los fumadores, por lo que las mujeres y niños están especialmente en riesgo de enfermedades relacionadas con la exposición al humo de tabaco. Recientemente, datos recogidos en más de 30 países -incluido México-, muestran que los niveles de nicotina en cabello de mujeres no fumadoras y sus hijos se incrementan con el número de fumadores en el hogar. ${ }^{20}$

Se conoce que la mayor exposición al humo de tabaco ocurre en los lugares de trabajo, donde las personas pasan muchas horas diariamente, ${ }^{21}$ por lo tanto, las leyes que recientemente se han aprobado en México son relevantes para la salud de todos los trabajadores. Además, estas leyes son necesarias no sólo en el contexto del control del tabaco, si no desde una perspectiva amplia de la salud pública. La epidemia de obesidad y diabetes que también enfrenta México, ${ }^{22}$ demanda intervenciones que protejan a la población de las consecuencias dañinas causadas por fumar y relacionadas con el peso, la distribución de la grasa corporal y la resistencia a la insulina. ${ }^{23}$

\section{Artículo 8 del CMCT}

El CMCT es el primer tratado internacional de salud pública elaborado bajo el auspicio de la $\mathrm{OMS}^{24}$ y México estuvo entre los primeros países en ratificarlo, el 28 de mayo de 2004. El CMCT propone una agenda global para enfrentar una epidemia que se ha extendido a todos los países. Para ello se propone una serie de medidas encaminadas a reducir la oferta y la demanda de tabaco, a la vez que se estimula la cooperación entre las partes. Especialmente relevante para el impulso que ha tomado México en materia legislativa es el "Artículo 8. Protección contra la exposición al humo de tabaco", que textualmente plantea:

"1. Las Partes reconocen que la ciencia ha demostrado de manera inequívoca que la exposición al humo 
de tabaco es causa de mortalidad, morbilidad y discapacidad.

2. Cada Parte adoptará y aplicará, en áreas de la jurisdicción nacional existente y conforme determine la legislación nacional, medidas legislativas, ejecutivas, administrativas y/u otras medidas eficaces de protección contra la exposición al humo de tabaco en lugares de trabajo interiores, medios de transporte público, lugares públicos cerrados y, según proceda, otros lugares públicos, y promoverá activamente la adopción y aplicación de esas medidas en otros niveles jurisdiccionales."

La protección de los no fumadores a través de la creación de espacios 100\% libres de humo de tabaco no sólo es la solución correcta, sino también es una solución posible. La aprobación de una ley federal y de una local en este mismo año, son la concreción de los compromisos adquiridos al ratificar el CMCT. Las barreras que puedan encontrar estas leyes para su implementación ya han sido derribadas en otros países y México puede aprender de esas experiencias. En las siguientes secciones examinamos los principales beneficios obtenidos de estas medidas y se desmienten los principales argumentos utilizados por la industria tabacalera.

\section{Reducción del consumo y beneficios para la salud}

Un primer efecto positivo -que por sí mismo justifica la intervención- es que las leyes que promueven los espacios sin humo de tabaco traspasan el derecho de propiedad sobre aire ambiental de los fumadores hacia los no fumadores y reducen la aceptación de fumar como conducta socialmente establecida. ${ }^{25}$ Pero sobre todo, la creación de espacios $100 \%$ libres de humo de tabaco es una medida efectiva porque reduce la prevalencia de consumo de tabaco, el número promedio de cigarrillos por día y promueven la cesación. ${ }^{26}$ Lo anterior se logra cuando se vela estrictamente por el cumplimiento de la ley; si sólo existe una legislación fuerte que se cumple relajadamente, su impacto será prácticamente nulo.

Un metaanálisis que incluyó 26 estudios sobre el impacto de la prohibición de fumar en lugares de trabajo en EUA, Canadá, Australia y Alemania concluye inequívocamente que la medida no sólo protege a los no fumadores de la exposición al humo de tabaco, sino que también estimula a los fumadores a reducir su consumo. ${ }^{27}$ Existe una gran diferencia en el impacto que se logra con restricciones totales a cuando sólo existe una restricción parcial. Se ha estimado que donde existe una legislación integral y sobre todo, que se vela por su cumplimiento, puede reducir el consumo de cigarrillos hasta 4.8 paquetes por persona por año. ${ }^{28}$

Un análisis econométrico del impacto de la prohibición de fumar en lugares de trabajo mostró que se puede reducir la prevalencia de fumadores entre 4 y $6 \%$, además de que el consumo diario de cigarrillos se redujo 10 por ciento. ${ }^{29}$ Estos impactos fueron diferentes entre empleados que trabajaban la jornada completa bajo esas condiciones y otros que sólo trabajaban parcialmente en espacios libres de humo de tabaco. Una intervención comunitaria examinó una cohorte de fumadores entre 1988 y 1993 y encontró que entre los trabajadores expuestos a restricciones para fumar en sus lugares de trabajo fue 1.25 veces más probable que hubieran intentado seriamente dejar de fumar, además de que un $25 \%$ más había logrado dejar el cigarrillo. ${ }^{30}$

Datos de estudios australianos también han mostrado reducciones de hasta 5.2 cigarrillos por día entre fumadores que trabajan en condiciones donde no se les permite fumar en el lugar de trabajo. ${ }^{31}$ Además del impacto en los trabajadores fumadores, desde los años noventa se ha visto en EUA que este tipo de medidas también influye en otros segmentos de la población. Por ejemplo, fuertes restricciones estatales o locales para fumar en lugares públicos también resultan en la reducción del consumo de cigarrillos entre adolescentes; además de que se observó una disminución en el número promedio de cigarrillos por día. ${ }^{32}$

Los ejemplos antes descritos dan cuenta del impacto positivo en la reducción del consumo cuando se aplican leyes que promueven los espacios 100\% libres de humo de tabaco. Sin embargo, también hay evidencia sólida de beneficios para la salud asociados con la aplicación de este tipo de medias. Por ejemplo, se reportó $40 \%$ de reducción en el número de hospitalizaciones por infarto agudo del miocardio tras seis meses de entrada en vigor de una ley que prohíbe fumar en lugares públicos y lugares de trabajo en una pequeña comunidad, Helena, del estado de Montana, EUA. La comparación se hizo respecto del número de hospitalizaciones en los mismos meses del año anterior a la entrada en vigor de la ley. ${ }^{33}$ Ese resultado estimuló otros estudios para confirmar un resultado de tan grandes implicaciones en términos de salud pública. Una investigación en Pueblo, otra comunidad pequeña en el estado de Colorado, EUA, analizó las hospitalizaciones por infarto agudo del miocardio registradas un año y medio antes y un año y medio después de la entrada en vigor de una ley similar. ${ }^{34}$ También encontró una reducción significativa en el número de hospitalizaciones por infarto, (riesgo relativo $(R R)=0.73$, con intervalo de confianza $(I C)$ de $95 \%, 0.63$ a 0.85 . Otras comunidades aledañas, pero sin ley de prohibición de fumar, no registraron una reducción 
estadísticamente significativa, $R R=0.85$ (IC95\% 0.63-1.16) y $R R=0.97$ (IC95\% 0.89-1.06).

El mismo beneficio ha sido reportado en otros contextos. Por ejemplo, un estudio italiano encontró una reducción de $11 \%$ en las hospitalizaciones por infarto agudo del miocardio después de la entrada en vigor de una prohibición de fumar (razón ajustada de tasas 0.89, con IC95\% 0.81-0.98). ${ }^{35}$ En el estado de Nueva York, EUA, la prohibición total de fumar entró en vigencia en julio de 2003 y el análisis de las estadísticas hospitalarias de 2004 reportó una reducción de 8\% en las hospitalizaciones por infarto agudo del miocardio; derivado de tal decremento, se estima un ahorro de 56 millones de dólares de costos directos de atención médica. ${ }^{36}$

Otra ventaja es la disminución de síntomas agudos en trabajadores, pues los beneficios respiratorios asociados con la prohibición de fumar en lugares de trabajo son inmediatos. Las medidas aplicadas en bares y restaurantes de California desde hace más de una década se asocian con reducción a la exposición al humo de tabaco, que a su vez se asocia con la reducción de síntomas de irritación en ojos, nariz y garganta y con síntomas respiratorios, tales como tos, estornudos y falta de aire. ${ }^{37}$ Otro artículo publicado en 2006 muestra que la prohibición de fumar en lugares de trabajo en Escocia condujo a la reducción significativa en los síntomas atribuibles a la exposición al humo de tabaco en trabajadores de bares, que además mejoraron su función pulmonar. ${ }^{38}$ Otros parámetros indicativos de inflamación celular también se vieron reducidos, así como los niveles de cotinina en suero. Los trabajadores asmáticos mejoraron su calidad de vida y se redujeron sus problemas causados por inflamación de las vías respiratorias.

\section{Acciones de un país en desarrollo}

La epidemia de tabaquismo es un fenómeno creciente entre las naciones en desarrollo. ${ }^{39}$ En el caso de México, datos de la más reciente Encuesta Nacional de Adicciones indican que $26 \%$ de la población mexicana adulta está expuesta al humo de otros fumadores. ${ }^{40}$ Estas cifras son consistentes con lo reportado por la Encuesta de Tabaquismo y Juventud 2006, donde 57.2\% de los adolescentes reportaron que otras personas fuman en su presencia en lugares públicos. ${ }^{5}$ Los niveles de nicotina en el aire detectados en lugares públicos y de trabajo en la Ciudad de México, ${ }^{41}$ así como las elevadas concentraciones de cotinina en orina de no fumadores que asistieron a discotecas ${ }^{42}$ son pruebas de que sí es necesario proteger a los no fumadores mexicanos a través de leyes que promuevan los espacios $100 \%$ libres de humo de tabaco.
En el ámbito mundial existen diferencias en la prevalencia y alcance de las leyes que promueven los espacios libres de humo de tabaco. Los pasos que recientemente ha dado México en materia legislativa para proteger la salud de los no fumadores lo colocan en una posición muy destacada, especialmente entre las naciones en desarrollo. Por ejemplo, según el reciente Reporte MPOWER presentado por la OMS, sólo 26\% de los estados miembros tienen una legislación moderada o completa respecto del derecho de respirar aire limpio del humo de tabaco. ${ }^{43}$ Conociendo que no existe ningún nivel de exposición que no sea dañino para la salud, la única intervención que realmente protege a la gente es la prohibición total de fumar en todos los espacios cerrados. Sin embargo, esta medida que parece tan obvia desde el punto de vista de salud pública y es ampliamente popular,* encuentra resistencias para su implementación.

\section{Argumentos de la industria tabacalera}

La industria tabacalera ha querido evitar esta medida y para ello ha recurrido a todo tipo de ardides, el más viejo truco es alimentar la controversia sobre si realmente el humo de tabaco es dañino. ${ }^{17}$ Ya desde hace muchos años también nos ha querido distraer con propuesta de intervenciones inefectivas. ${ }^{44}$ Más encubiertamente, usa la voz de los propietarios de negocios y alimenta el temor de la catástrofe económica asociada con esta medida -ya sea por pérdida de clientes o por lo caro que resulta crear un espacio libre de humo-. La industria también mueve hilos dentro del tejido social, estimulando y dando apoyo a agrupaciones de fumadores que argumentan que se les está discriminando. ${ }^{45}$ Según las noticias que han aparecido en los medios, de todo esto ha habido un poco en México en los últimos meses.

La OMS propone un proceso paso a paso como el más efectivo para crear ambientes libres de humo de tabaco. ${ }^{46}$ Según este enfoque, un primer paso que deben emprender los gobiernos es educar a la población sobre los daños de la exposición al humo de tabaco. Cuando se ha elevado el nivel de concientización sobre la materia,

\footnotetext{
* Según el reporte MPOWER de la OMS, ${ }^{43}$ el apoyo a la prohibición total de fumar en bares y restaurantes es muy alto en lugares reconocidos por tener legislación muy fuerte: superior a $60 \%$ en Nueva Zelandia, más de $70 \%$ en el estado de California y la ciudad de Nueva York; $80 \%$ en Uruguay y más de 90\% en Irlanda. En México, 87\% de los estudiantes de secundaria apoya la prohibición de fumar en lugares públicos. ${ }^{5}$
} 
hay buenas condiciones para legislar al respecto. Las leyes de promoción de espacios libres de humo de tabaco deben ir acompañadas de claras medidas para velar por su cumplimiento y disponer de sanciones para quienes violen lo dispuesto. La vigilancia por el cumplimiento es el mayor reto y debe ser compartido por el estado, cuando vela por sus leyes, y por la ciudadanía, cuando defiende su derecho a respirar aire limpio.

En el centro del debate generado por las leyes para crear espacios 100\% libres de humo en México hay varios temas bien conocidos. Uno es el temor a las pérdidas económicas por disminución de clientes; otro es sobre lo injusta que resulta la medida para los pequeños negocios y, relacionado con esto ultimo, se ha mencionado la posibilidad de aplicar medidas menos drásticas. Un cuarto tema, a nuestro juicio, es el relativo al derecho de los fumadores. Muchos estudios nacionales y locales demuestran que no hay pérdidas económicas asociadas con la implementación de leyes de protección a los no fumadores. Está muy documentado lo sucedido en Noruega ${ }^{47}$ y en California, ${ }^{48}$ respecto de que crear espacios libres de humo de tabaco no disminuyó los ingresos del sector de servicios, específicamente bares, restaurantes y hotelería. En el contexto latinoamericano, tenemos evidencia de un estudio econométrico en Uruguay de que la prohibición de fumar no afectó la recaudación en restaurantes y parrilladas en los meses siguientes a la entrada en vigor de la ley en 2006.* Tampoco se ha demostrado que la prohibición de fumar afecte a los pequeños negocios, de hecho, datos de la encuesta Zagat demuestran que $96 \%$ de las personas en la ciudad de Nueva York van a comer afuera tanto, o más que antes, de que se aplicara la ley. ${ }^{49}$ Además, la misma encuesta reporta que los restaurantes y bares de la ciudad se han beneficiado económicamente..$^{50}$

También se ha dicho que va en detrimento de los pequeños negocios exigir la remodelación de los locales con barreras físicas, para que realmente las áreas de no fumar estén libres de humo. Entendemos que ésta es una preocupación legítima por parte de los propietarios, sin embargo, otras medidas como sistemas de ventilación o extracción de aire definitivamente no funcionan. La Sociedad Americana de Ingenieros de Calefacción, Refrigeración y Aire Acondicionado (ASHRAE, por sus siglas en inglés) fue consultada y concluyó al respecto que: "En la actualidad, la única forma de eliminar efectivamente

\footnotetext{
* Comunicación personal con la Dra. Adriana Blanco de la OPS, quien facilitó el documento no publicado: Ramos A, Curti D. Evaluación económica del impacto de la medida de 100\% libre de humo de tabaco sobre el sector de la hostelería en Uruguay. Montevideo, marzo de 2007.
}

los riesgos a la salud asociados con exposición al humo de tabaco en interiores es prohibiendo fumar". ${ }^{51} \mathrm{El}$ debate de cómo no afectar a los pequeños propietarios ya se dio en Uruguay y la mejor solución, tanto desde el punto de vista de salud pública, como para no afectar la capacidad de competencia de ningún negocio, fue la prohibición de fumar en todos los lugares públicos, incluyendo bares, restaurantes y casinos a partir de marzo de 2006. ${ }^{52}$

Otro reto por atender cuando se aprueban leyes que promueven los espacios $100 \%$ libres de humo de tabaco viene de la población fumadora. Ciertamente, éste es un asunto controvertido que es aprovechado tanto por los cabilderos de la industria, como por grupos de confrontación que se manifiestan en defensa del derecho de los fumadores. ${ }^{45}$ De un lado, los fumadores argumentan que existe discriminación hacia ellos y que el gobierno se excede al interferir con un comportamiento individual que involucra el uso de una sustancia legal. Por otro lado están los no fumadores, quienes además de ser mayoría tienen el derecho a respirar un aire limpio sin exponerse a los riesgos asociados con el humo de tabaco y que sólo pueden ejercer ese derecho cuando por ley se delimita dónde se puede fumar y dónde no. A manera de respuesta a esta discusión queremos citar las palabras de C. Everett Koop, Surgeon General de los Estados Unidos durante el periodo 1982-1989, que en el prefacio de uno de los reportes más influyentes en el tema de fumar involuntariamente escribió: "El derecho de los fumadores a fumar termina cuando su conducta afecta la salud y el bienestar de los otros; además, es responsabilidad de los fumadores asegurarse de que ellos no están exponiendo a los no fumadores a los efectos potencialmente dañinos del humo de tabaco".$^{10}$

\section{Apoyo del IGTC a México}

El Artículo 20 del CMCT se refiere a la cooperación técnica y científica para la investigación, vigilancia e intercambio de información. Un buen ejemplo de ello es la estrecha colaboración con México que ha desarrollado el Instituto para el Control Global del Tabaco de la Escuela de Salud Pública de la Universidad Johns Hopkins (IGTC, por sus siglas en inglés). Desde hace una década el IGTC ha apoyado los esfuerzos del Instituto Nacional de Salud Pública por impulsar el control del tabaco en el país. Esa colaboración ha sido documentada con anterioridad. ${ }^{53}$ Del apoyo técnico y financiero han resultado varias publicaciones conjuntas en las que se abordan temas como exposición al humo de tabaco en hogares ${ }^{20,54}$ y en lugares públicos ${ }^{41}$ creación de infraestructura para el control del tabaco, ${ }^{53}$ consumo de tabaco en adolescentes $^{55}$ y medición de biomarcadores de nicotina en 
fumadores. ${ }^{56,57}$ Asimismo, se ha apoyado el arranque del laboratorio analítico de compuestos del tabaco y se ha dado entrenamiento a varios investigadores.

Nuestro curso de verano se ha desarrollado desde 2000 y han participado tomadores de decisiones y activistas del control del tabaco de diferentes niveles y de las distintas entidades federativas, especialmente representantes de los consejos estatales Contra las Adicciones. El curso se desarrolla conjuntamente con expertos nacionales y ha contribuido positivamente a fortalecer la capacidad de México para el control del tabaco. Capacidad nacional para el control del tabaco se refiere a la capacidad del país para desarrollar y emprender acciones multisectoriales e integrales que faciliten las estrategias adecuadas de prevención y control que reduzcan el consumo de tabaco. Lo anterior se refleja en tres componentes esenciales: 1 ) conocimientos y liderazgo, 2) infraestructura y 3) evidencia empírica. ${ }^{58}$ Las leyes antitabaco aprobadas recientemente dan muestra de la madurez que ha alcanzado México en su programa de control.

En 2007 el curso de verano extendió su formato y compartió espacio con otras organizaciones nacionales e internacionales que se reunieron en un taller de abogacía con los principales actores del control del tabaco en el país a diferentes niveles. De igual forma, el IGTC también ha expresado firmemente su opinión respetuosa ${ }^{59}$ cuando las acciones para el control del tabaco en México se han alejado de lo propuesto por el CMCT. Recientemente, durante el proceso de discusión de las dos leyes que protegen a los no fumadores, las autoridades de salud del país invitaron a expertos del IGTC para que dieran su testimonio ante los tomadores decisiones. La fructífera colaboración que ha existido, las metas comunes y los nuevos retos por extender las fronteras del control del tabaco en todo del mundo, nos permiten decir que la colaboración del IGTC con México continuará en el futuro.

\section{Comentarios finales}

Las leyes que se han aprobado en los últimos meses en México en materia de protección a los no fumadores reflejan sin duda el compromiso del gobierno en su lucha por el control del tabaquismo en el país. Una legislación fuerte que promueve los espacios 100\% libres de humo es difícil de conseguir sin el consenso de las fuerzas políticas, el interés de las autoridades sanitarias, el apoyo de la sociedad civil y la participación de instituciones académicas y de investigación. Todo ello habla por sí mismo del ambiente propicio para el control del tabaco que se vive en México hoy. Sin embargo, los retos van más allá de la aprobación de leyes fuertes, ya que la industria tabacalera es muy poderosa y continuará insistiendo en sus propósitos.

Lograr implementar cuanto antes las medidas acordadas, velar por su cumplimiento, evaluar su impacto, así como desmentir y desalentar otro tipo de iniciativas ajenas a la prohibición total de fumar en lugares cerrados, son algunos de las tareas inminentes. También es necesario seguir promoviendo iniciativas de leyes locales, seguir usando la política fiscal para desalentar el consumo, promover la cesación y prevenir el consumo entre los jóvenes. Todas esas acciones son más efectivas cuando se aplican conjuntamente para producir el efecto sinérgico de un verdadero programa exhaustivo. ${ }^{61}$ En el reporte que presentó la OMS recientemente sobre la situación mundial de la epidemia de tabaquismo se recomiendan seis acciones: 1) monitorear la prevalencia de fumadores y las políticas de prevención; 2) proteger a la gente del humo de tabaco; 3) ofrecer ayuda para dejar de fumar; 4) advertir sobre los daños que causa el tabaco; 5) reforzar las prohibiciones a la publicidad, promoción y patrocinio de la industria tabacalera y 6) aplicar impuestos a los productos de tabaco. ${ }^{43} \mathrm{En}$ cierta forma todas esas acciones ya están iniciadas en México. El primer país del hemisferio occidental que ratificó el CMCT ahora debe ejecutar cabalmente su agenda para combatir el tabaquismo, y la legislación es un elemento clave para ello.

\section{Agradecimientos}

La preparación de este artículo fue posible gracias al apoyo de Fogarty International Center of the National Institutes of Health de Estados Unidos a través del financiamiento No. R01-HL-73699. También reconocemos el apoyo que recibimos de Bloomberg Initiative to Reduce to Tobacco Use a través del financiamiento No. 906400. Raydel Valdés-Salgado dispone de una beca parcial otorgada por la OPS para cursar estudios de doctorado, durante los cuales ha sido redactado este artículo.

\section{Referencias}

I. Ley General para el Control del Tabaco. Aprobada por el Senado de la República. México, 4 de marzo de 2008.

2. Ley de Protección a la Salud de los No Fumadores en el Distrito Federal. Aprobada por la Asamblea Legislativa del Distrito Federal el 26 de febrero y publicada en la Gaceta Oficial del Distrito Federal el 4 de marzo de 2008, México, 2008.

3. Ley del Impuesto Especial sobre Producción y Servicios. Diario Oficial de la Federación, miércoles 27 de diciembre de 2006. Cuarta sección. México, 2006:80.

4. Valdés-Salgado R. Las cifras de la Epidemia. Daños a la salud y mortalidad atribuible. En Valdés-Salgado R, Lazcano Ponce EC, Hernández- 
Ávila M, eds. Primer Informe sobre el combate al tabaquismo. México ante el Convenio Marco para el Control del Tabaco. México: Instituto Nacional de Salud Pública, 2005:29-4I.

5. Valdés-Salgado R, Reynales-Shigematsu LM, Lazcano-Ponce E, Hernández-Ávila M. Antes y después del Convenio Marco en México: una comparación desde la Encuesta sobre Tabaquismo en Jóvenes 2003 y 2006. Salud Publica Mex 2007;49(2): I55-II6.

6. Guerin MR, Jenkins RA, Tomkins BA. The chemistry of environmental tobacco smoke: composition and measurement. Chelsea, Michigan: Lewis Publishers Inc, 1992.

7. Hirayama T. Non-smoking wives of heavy smokers have a higher risk of lung cancer: a study from Japan. BMJ I98I;282:183-185.

8. Cameron P, Kostin JS, Zaks JM, Wolfe JH, Tighe G, Oseletf B, et al. The health of smokers' and nonsmokers' children. Journal of Allergy 1969;43(6):336-341.

9. US Department of Health, Education, and Welfare. The health consequences of smoking: A Report of the Surgeon General: 1972. Washington: US Department of Health, Education, and Welfare, Public Health Services and Mental Health Administration, 1972. DHEW Publication No. (HSM) 72-75I6.

10. US Department of Health and Human Services. The health consequences of involuntary smoking: A Report of the Surgeon General. Rockville (MD): US Department of Health and Human Services. Public Health Service, Centers for Disease Control, Center for Health Promotion, Office on Smoking and Health; 1986. DHHS Publication No. (CDC) 87-8398.

II. US Environmental Protection Agency. Respiratory Health Effects of Passive Smoking: Lung Cancer and Other Disorders. Washington: US Environmental Protection Agency, Office of Research and Development, Office of Air Radiation, 1992. Report No. EPA/600/6-90/0006F.

12. Scientific Committee on Tobacco and Health. Report of the Scientific Committee on Tobacco and Health. London: The Stationery Office, 1998. 13. California Environmental Protection Agency. Health Effects of Exposure to Environmental Tobacco Smoke. Office of Environmental Health Hazards Assessment. Sacramento, California Environmental Protection Agency, 1997.

14. World Health Organization. International Consultation on Environmental Tobacco Smoke (ETS) and Child Health. Consultation Report. Geneva: WHO, 1999.

15. International Agency for Research on Cancer. IARC Monograph on the Evaluation of Carcinogenic Risk to Humans: Tobacco Smoke and Involuntary Smoking. Vol. 83 Lyon (France): International Agency for Research on Cancer, 2004.

16. US Department of Health and Human Services. The health consequences of involuntary exposure to tobacco smoke: A Report of the Surgeon General. Atlanta (GA): US Department of Health and Human Services. Centers for Disease Control and Prevention. National Center for Chronic Disease Prevention and Health Promotion, Office on Smoking and Health, 2006.

17. Aguinaga Bialous S, Shatenstein S: PROFITS OVER PEOPLE. Tobacco Industry Activities to Market Cigarettes and Undermine Public Health in Latin America and the Caribbean. Washington DC: Pan American Health Organization, November 2002.

18. US Department of Health and Human Services (USDHHS). The health consequences of smoking - chronic obstructive lung disease. A report of the Surgeon General. Washington, DC: US Government Printing Office, 1984.

19. California Environmental Protection Agency (Cal EPA) and Air Resources Board. Proposed identification of environmental tobacco smoke as a toxic air contaminant. Sacramento, CA, California Environmental Protection Agency, 2005.

20. Wipfli H, Avila-Tang E, Navas-Asien A, Sungroul K, Onicescu G, Yuan J, Breysse P, Samet JM: Secondhand smoke exposure among women and children: evidence from 3 I counries. Am J Public Health 2008;98(4):672-679.
21. Eisner MD: Editorial. Banning smoking in public places. JAMA 2006;296(14): 1778-1779.

22. Rivera JA, Barquera S, Gonzalez-Cossio T, Olaiz G, Sepulveda J: Nutrition transition in Mexico and in other Latin American countries. Nutr Rev 2004;62(II):S149-S157.

23. Chiolero A, Faeh D, Paccaud F, Cornuz J: Consequences of smoking for body weight, body fat distribution, and insulin resistance. Am J Clin Nutr 2008;87(4):801-809.

24. The WHO Framework Convention on Tobacco Control, World Health Assembly Resolution 56. I, May 21, 2003. Disponible en: http:// www.paho.org/Spanish/DD/PUB/sa56rl.pdf [consultado 17 abril 2008]. 25. National Cancer Institute. Health effects of exposure to environmental tobacco smoke: the report of the California Environmental Protection Agency. Bethesda, MD. National Cancer Institute, 1999 (Smoking and Health Monograph 10).

26. Jha P, Chaloupka F, eds. Tobacco Control in Developing Countries. Oxford University Press Inc., New York.

27. Fichtenberg CM, Glantz SA: Effect of smoke-free workplace on smoking behaviour: systematic review. BMJ 2002; 325:188.

28. Yurekli A, Zhang P. The impact of clean indoor-air laws and cigarette smuggling on demand for cigarettes: an empirical model. Health Econ 2000;9(2):159-170.

29. Evans WN, Farrelly MC, Montgomery E. Do workplace smoking bans reduce smoking? American Economy Review 1999;89(4):728-747. 30. Glasgow RE, Cumming KM, Hyland A: Relationship of worksite smoking policy to changes in employee tobacco use: findings form COMMIT. Tob Control 1997;6(suppl 2):S44-S48.

31. Borland R, Chapman S, Owen N, Hill D: Effects of workplace smoking bans on cigarette consumption. Am J Public Health 1990;80(2): 178-180.

32. Chaloupka FJ, Wechsler H: Price, tobacco control policies and smoking among young adults. J Health Econ 1997;16(3):359-373.

33. Sargent RP, Shepard RM, Glantz SA. Reduced incidence of admissions for myocardial infarction associated with public smoking ban: before and after study. BMJ 2004;328:977-983.

34. Bartecchi C, Alsever RN, Nevin-Woods C, Thomas WM, Estacio $\mathrm{RO}$, Bartelson BB, et al. Reduction in the Incidence of Acute Myocardial Infarction Associated With a Citywide Smoking Ordinance. Circulation 2006; I 14:1490-1496.

35. Barone-Adesi F, Vizzini L, Merletti F, Richiardi L: Short-term effects of Italian smoking regulation on rates of hospital admission for acute myocardial infarction. Eur Heart J 2006;27:2468-2472.

36. Juster HR, Loomis BR, Hinman TM, Farrelly MC, Hyland A, Bauer UE, et al. Declines in Hospital Admissions for Acute Myocardial Infarction in New York State After Implementation of a Comprehensive Smoking Ban. Am J Public Health 2007;97:2035-2039.

37. Eisner MD, Smith AK, Blanc PD: Bartenders' Respiratory Health after Establishment of Smoke-Free Bars and Taverns. JAMA 1998;280:1909-19|4. 38. Menzies D, Nair A, Williamson PA, Schembri S, Al-Khairalla MZH, Barnes M, et al. Respiratory Symptoms, Pulmonary Function, and Markers of Inflammation Among Bar Workers Before and After a Legislative Ban on Smoking in Public Places. JAMA 2006;298: 1742-1746.

39. The World Bank: Curbing the Epidemic. Governments and the Economics of Tobacco Control. Washington DC, 1999. 40. Kuri-Morales PA, González-Roldán JF, Hoy MJ, Cortés-Ramírez M. Epidemiología del tabaquismo en México. Salud Publica Mex 2006;48(supl I):S9I-S98.

4I. Barrientos-Gutiérrez T, Valdés-Salgado R, Reynales-Shigematsu LM, Navas-Acien A, Lazcano-Ponce. Exposición involuntaria al humo de tabaco en lugares públicos de la Ciudad de México. Salud Publica Mex 2007;49(2):205-212.

42. Lazcano-Ponce E, Benowitz N, Sánchez-Zamorano LM, BarbosaSanchez L, Valdés-Salgado R, Jacob III P, et al. Secondhand smoke exposure in Mexican discotheques. Nicotine Tob Res 2007;9(10):1021-1026. 
43. WHO Report on the Global Tobacco Epidemic. The MPOWER package. Geneva, World Health Organization 2008. Disponible en: http://www.who.int/tobacco/mpower/mpower_report_full_2008.pdf [consultado 17 abril 2008].

44. Sebrie EM, Glantz SA. "Accommodating" smoke-free policies: tobacco industry's Courtesy of Choice programme in Latin America. Tob Control. 2007; 16(5):e6.

45. Salojee Y, Dagli E: Tobacco industry tactics for resisting public policy on health. Bulletin of the World Health Organization, 2000, 78 (7): 902-910. 46. World Health Organization Tobacco Free initiative: Building blocks for tobacco control: a handbook. Geneva: WHO, 2004.

47. The Smoke Free Partnership: Lifting the smokescreen - 10 reasons for a smoke free Europe. Belgium: ERSJ Itd., 2006.

48. Glantz SA. Effect of smokefree bar law on bar revenues in California.

Tob Control 2000; 9: I II-II2.

49. Rudin A. Zagat 2004 New York City restaurant survey finds local dining economy in comeback mode; 2936 I voters turn out for Zagat's $25^{\text {th }}$ annual NY guide. Press release, 20 October 2003.

50. Campaign for Tobacco-Free Kids. Zagat restaurant survey provides more evidence that New York City's smoke-free law is not hurting business. Press release, 21 October 2003.

51. American Society of Heating, Refrigerating and Air-Conditioning Engineers. Position Document on Environmental Tobacco Smoke. Approved by ASHRAE Board of Directors June 30, 2005. Disponible en: http://www.ashrae.org/docLib/200582 I I239_347.pdf [consultado 17 abril 2008].

52. Decreto del Poder Ejecutivo $N^{\circ}$ I68/005 de 3I de mayo de 2005. Decreto que prohíbe fumar en lugares públicos a partir del $1^{\circ}$ de marzo de 2006. Montevideo, Uruguay, 2005.
53. Stillman F, Yang G, Figueiredo V, Hernandez-Avila M, Samet J. Building capacity for tobacco control research and policy. Tob Control 2006;15; 18-23.

54. Barrientos-Gutiérrez T, Reynales-Shigematsu LM, Avila-Tang E, Wipfli H, Lazcano-Ponce E. Exposición al humo de tabaco en hogares de la Ciudad de México: análisis de nicotina ambiental y en cabello de niños y mujeres. Salud Publica Mex 2007;49(supl 2):S224-S232.

55. Arillo-Santillan E, Lazcano-Ponce E, Hernandez-Avila M, Fernandez E, Allen $B$, Valdes $R$, et al. Association between individual and contextual fators and smoking in 13,293 Mexican students. Am J Prev Med 2005;28(I):4I-5I.

56. Campuzano JC, Hernandez-Avila M, Jaakkola MS, Lazcano-Ponce E, Kuri-Morales $P$, Bautista $P$, et al. Determinants of salivary cotinine levels among current smokers in Mexico. Nicotine Tob Res 2004;6(6):997-1008. 57. Blackford A, Yang G, Hernandez-Avila M, Przewozniak K, Zatonski $W$, Figueiredo $V$, et al. Cotinine concentration in smokers from different countries: Relationship with amount smoked and cigarette type. Cancer Epidemiol Biomarkers Prev 2006; I5(I0): 1799-1804.

58. Wipfli H, Stillman F, Tamplin S, Da Costa e Silva VL, Yach D, Samet JM. Achieving the Framework Convention on Tobacco Control's potential by investing in national capacity. Tob Control 2004; I3;433-437.

59. Samet J, Wipfli H, Perez-Padilla R, and Yach D. Mexico and the tobacco industry: doing the wrong thing for the right reason? BMJ 2006;332:353-354. 60. Centers for Disease Control and Prevention. Best Practices for Comprehensive Tobacco Control Programs - 2007. Atlanta: US Department of Health and Human Services, Centers for Disease Control and Prevention, National Center for Chronic Disease Prevention and Health Promotion, Office on Smoking and Health, October 2007. 